\title{
Artigos
}

0

\section{IL ‘VOLONTARIATO’ DEI RICHIEDENTI ASILO IN ITALIA}

\author{
The 'volunteering' of asylum seekers in Italy
}

Martina Pasqualetto ${ }^{1}$

\begin{abstract}
Riassunto. L'articolo analizza la politica pubblica italiana che prevede il coinvolgimento dei richiedenti asilo in attività di 'volontariato' a favore delle amministrazioni locali per calmare il malcontento popolare causato dalla loro presunta inattività. In primo luogo l'autrice mette in discussione I'efficacia di tale policy e ne destruttura il discorso governativo mettendone in luce le mistificazioni. Mediante una ricerca esplorativa ispirata alla grounded theory si dimostra inoltre che la reale natura delle attività promosse risiede nel lavoro gratuito e che esse veicolano un messaggio di simbolica e percepita utilità dei profughi che impatta negativamente sull'immaginario collettivo rispetto al tema del diritto d'asilo.
\end{abstract}

Parole chiave: richiedenti asilo, politiche pubbliche, volontariato, lavoro gratuito.

\begin{abstract}
The article analyzes the Italian public policy encouraging 'volunteering' activities of asylum seekers in favor of local administrations, whose aim is to spread welcoming feelings among the population acting against their contested passivity. Firstly the author discusses the efficiency of the policy and debuilds the governmental discourse enlightening its mystifications. Secondly, through an explorative on-field research inspired by the grounded theory method it is shown that the real nature of the promoted activities lies in unpaid work, as well as they convey a symbolic and perceived 'asylum-seekers-utility' message which has a negative impact on the public consciousness regarding the asylum issue.
\end{abstract}

Keywords: asylum seekers, public policies, volunteering, unpaid work.

1 Centro di Ricerca ESRU (European Social Unit Research), Università di Barcellona. Mestre, Venezia, Italia. 


\section{Introduzione}

L'esponenziale incremento degli arrivi di richiedenti asilo in Italia, causato dai conflitti medio-orientali e dalle perduranti crisi economiche, ambientali e umanitarie nei paesi africani, ha posto il tema della cosiddetta 'emergenza profughi' al centro dell'agenda politica nazionale. All'interno delle politiche pubbliche di accoglienza predisposte dai Ministeri competenti un ruolo di particolare importanza è stato assegnato ai Comuni. Quest'ultimi, in quanto entità politiche più vicine al cittadino, sono stati investiti del compito strategico di mediare tra una popolazione generalmente maldisposta all'accoglienza e la crescente presenza dei richiedenti asilo nei territori.

I Comuni italiani vivono però un periodo di forte difficoltà economica a causa del Patto di Stabilità Interno per il controllo della spesa pubblica e del blocco del trasferimento di risorse statali ${ }^{2}$. Per agevolare l'ordinaria gestione del territorio, il Governo ha dotato tali enti di strumenti legislativi che incoraggiano i privati cittadini a fornire gratuitamente prestazioni lavorative a favore della comunità, sulla scorta di un nuovo discorso sulla 'partecipazione attiva' o in forma sostitutiva di debiti con la pubblica amministrazione: nell'ordinamento giuridico italiano sono dunque apparse forme istituzionalizzate di lavoro gratuito. A seguito delle trasformazioni economiche dovute all'affermarsi dell'ideologia e delle politiche neoliberiste, questa categoria è ormai trasversale ai settori pubblico e privato: importanti seppur sporadici contributi accademici, provenienti soprattutto dall'ambito anglosassone, costituiscono ad oggi una linea di ricerca che circoscrive e discute la crescente riconfigurazione in termini di lavoro gratuito di attività e prestazioni che popolano tanto il mondo della formazione (è di grande attualità il dibattito sugli stage non retribuiti, ad esempio) ${ }^{3}$, quanto quello del volontariato ${ }^{4}$.

Rientra in quest'ultimo ambito la recente politica pubblica italiana denominata 'volontariato dei richiedenti asilo', la quale ne prevede il coinvolgimento in attività di pubblica utilità a favore dei Comuni con il proclamato obiettivo di facilitare la loro accettazione da parte della popolazione e costruire percorsi di integrazione. Ufficializzata nel 2014 tramite circolare del Ministero degli Interni, nel discorso governativo e mediatico la proposta è

2 CGIA DI MESTRE. Regioni ed enti locali: tagliati 25 miliardi di trasferimenti. 2015. Disponibile su: <http://www.cgiamestre.com/articoli/22215>. Visitato il 30.12.2016.

3 Sul tema: RODINO-COLOCINO, Michelle, BEBERICK, Stephanie Nicholl. "You kind of have to bite the bullet and do bitch work". How internships teach students to unthink exploitation in public relations; HOPE, Sophie, FIGIEL, Joanna. Interning and investing: Rethinking unpaid work, social capital, and the "Human capital regime".

4 Ad esempio: MAES, Kenneth. Volunteerism or labor exploitation? Harnessing the volunteer spirit to sustain AIDS treatment programs in urban Ethiopia. 
stata ricondotta alla fuorviante categoria del Volontariato; al contrario, come lo studio intende dimostrare, essa va annoverata tra le forme istituzionalizzate di lavoro gratuito.

Conseguentemente all'assenza di materiale bibliografico sul tema, è stata condotta una ricerca esplorativa ispirata alla grounded theory $y^{5}$. Dopo una prima fase di raccolta ed esame degli atti governativi prodotti in relazione alla policy, dalla rassegna stampa nazionale e locale sul tema sono stati selezionati e analizzati due casi studio paradigmatici: il Comune di Torino e la località di Giazza di Selva di Progno, in provincia di Verona. In secondo luogo è stata implementata un'inchiesta sul campo nella provincia di Treviso, costituita da dieci interviste semi-approfondite ad amministratori comunali e operatori di strutture di accoglienza, testimoni a vario titolo dell'applicazione locale della policy. La ricerca è stata orientata da un duplice obiettivo: la prima fase di decostruzione del discorso governativo, mediante lo studio della politica stessa e l'osservazione delle modalità e delle motivazioni con la quale è stata implementata a livello locale, ha consentito in secondo luogo di avanzare ipotesi circa i reali natura, scopi ed impatto sull'opinione pubblica della politica in esame.

\section{Carenze e conseguenze di un inadeguato sistema d'accoglienza}

Il decreto legislativo n.142 del 18 agosto 2015 ha aggiornato la legislazione italiana sui richiedenti asilo, con l'obiettivo di adeguarla alle inedite caratteristiche quantitative e qualitative del fenomeno: negli ultimi cinque anni si è verificata una crescita esponenziale degli ingressi (dai circa 12mila del 2010 agli oltre 170 mila del 2016$)^{6}$, e si sono diversificate le provenienze e le traiettorie migratorie. I nuovi strumenti legislativi non si sono tuttavia dimostrati sufficienti a riformare un sistema d'accoglienza che presenta tutt'ora forti problematiche e rinfocola continue polemiche sulla presenza dei richiedenti asilo in Italia. La lunghissima permanenza dei profughi nelle strutture ad hoc in attesa dell'esame dell'istanza d'asilo' implica un lievitare, reale e percepito, della spesa pubblica dedicata all'accoglienza; persiste tuttavia un'endemica carenza di posti letto causata dal carattere emergenziale delle politiche messe in campo ${ }^{8}$. Lo scarso controllo sulla gestione delle risorse determina invece non

5 CHARMAZ, Kathy. Constructing grounded theory; STRAUSS, Anselm, CORBIN, Juliet. Basics of qualitative research.

6 MINISTERO DELL'INTERNO. I numeri dell'asilo. 2016. Disponibile su: <http://www.interno. gov.it/it/sala-stampa/dati-e-statistiche/i-numeri-dellasilo > . Visitato il 21.12.2016.

7 La permanenza dei profughi nei centri d'accoglienza può raggiungere i 12 mesi, a fronte dei 30 previsti dalla legge. FONDAZIONE LEONE MORESSA. La buona accoglienza. 2015. Disponibile su: <http://www.fondazioneleonemoressa.org/newsite/wp-content/uploads/2016/01/LABUON A- ACCOGLIENZA-20_01_2016.pdf>. Visitato il 21.12.2016.

8 NAGA ONLUS. (Ben)venuti! Indagine sul sistema di accoglienza dei richiedenti asilo a Milano e provincia. 2016. Disponibile su:<http://www.naga.it/tl_files/naga/(Ben)venuti_Naga.pdf $>$. 
rari episodi di malagestione, culminati nel caso giudiziario di 'Mafia Capitale' ${ }^{9}$. Per ciò che attiene alle prospettive di inserimento nella società, le carenze del sistema sono aggravate dalla generale situazione socio-economica: se secondo il decreto legislativo 142 i richiedenti asilo hanno la possibilità di lavorare dopo due mesi dalla presentazione dell'istanza, una serie di fattori (giuridici, economici, amministrativi, sociali) rendono questa possibilità perlopiù remota.

Quella che è ormai percepita come una 'sfida epocale' anima da mesi un dibattito pubblico accesissimo, mentre le innumerevoli manifestazioni di intolleranza popolare verificatesi a livello locale testimoniano la diffusione di un sentimento di paura e avversione nei confronti dei profughi. Lo confermano i dati di un recente sondaggio IPSOS: più della metà degli italiani è a favore della chiusura dei confini, il 70\% sospetta che tra i richiedenti asilo ci siano terroristi e più del $60 \%$ ritiene che molti di essi siano bogus ${ }^{10}$. Alla già presente diffidenza verso gli immigrati in generale, alimentata negli ultimi decenni da forze politiche e mezzi di informazione che ne hanno diffuso un'immagine mistificante e fuorviante ${ }^{11}$, si è aggiunta la rabbia diretta nello specifico verso i profughi. Una serie di notizie false e una generale mancanza di informazione circa i temi dell'asilo e della situazione geopolitica internazionale hanno trovato terreno fertile in un tessuto sociale scosso dalla crisi economica e dalle disuguaglianze sociali: come in altri paesi europei, anche in Italia il richiedente asilo rappresenta un capro espiatorio da additare come responsabile delle difficoltà che il Paese attraversa. Le montanti preoccupazione e rabbia manifestate dalla popolazione autoctona, spesso maldisposta a condividere le sempre più esigue risorse statali e gli spazi cittadini percepiti come sempre meno sicuri con questi nuovi abitanti, hanno spinto il Governo ad elaborare delle politiche pubbliche che impattassero direttamente sulla percezione da parte dei cittadini della presenza dei richiedenti asilo sul territorio.

\section{II 'volontariato' dei richiedenti asilo}

\section{Caratteristiche ed implementazione della policy}

Tra le varie colpe di cui i profughi sono accusati appare il loro costituire un peso economico, l'essere 'mantenuti' dallo Stato illegittimamente, il 'bighellonare' tutto il giorno godendo di vitto e alloggio a carico dei contribuenti.

Visitato il 21.12.2016.

9 IL SOLE 24 ORE. 'Mafia Romana', il business dei centri di accoglienza. 03.12.2014. Disponibile su:<http://www.ilsole24ore.com/art/notizie/2014-12-03/mafia-romana-business-centriaccoglienza--121834.shtml?uuid=ABNP7OLC $>$. Visitato il 21/12/2016.

10 IPSOS. Global Views on Immigration and the Refugee Crises. 2016. Disponibile su:<https://www. ipsos-mori.com/Assets/Docs/Polls/ipsos-global-advisor-immigration-and-refugees-2016-charts. pdf >. Visitato il 21.12.2016.

11 PEROCCO, Fabio. Trasformazioni globali e nuove disuguaglianze, p. 114-115. 
È con riferimento a questa presunta problematica che il 27 novembre 2014 viene emessa la circolare 14290, firmata dal Capo Dipartimento per l'Immigrazione e le Libertà Civili Mario Morcone e promossa pubblicamente dall'allora Ministro dell'Interno Angelino Alfano. In essa il Governo individua nell'inattività dei richiedenti asilo la causa principale dell'intolleranza popolare e predispone il coinvolgimento dei profughi "in attività [...] di pubblica utilità svolte a favore delle popolazioni locali [...] esclusivamente su base volontaria e gratuita", ritenendo in tal modo di assicurare loro "maggiori prospettive di integrazione nel tessuto sociale del nostro Paese, scongiurando un clima di contrapposizioni nei loro confronti". La circolare è stata implementata territorialmente mediante protocolli promossi dalle Prefetture, le emanazioni locali del Governo. Secondo i dati del Piano Accoglienza 2016, alla fine dell'anno precedente se ne contavano un centinaio: 7 regionali, 14 provinciali, e più di 70 comunali ${ }^{12}$. Dall'analisi dei protocolli rinvenuti in rete ${ }^{13}$ emerge una politica pubblica dal profilo trilaterale: le Prefetture, gli enti locali ed il Terzo Settore si impegnano ad intrattenere "rapporti di collaborazione per l'individuazione e la gestione di eventuali criticità", con rispettivi ruoli di coordinamento e controllo, proposta e promozione. Ad emergere come fondamentale è il coinvolgimento del Terzo Settore: i richiedenti asilo devono risultare iscritti ad una associazione di promozione sociale, regola che giustifica il ricondurre questa politica pubblica all'ambito giuridico del Volontariato. Ai profughi, destinatari formali dell'iniziativa governativa, è meramente riservata la possibilità di aderire in maniera "libera, volontaria e gratuita" all'associazione e di impegnarsi a "rendere una o più prestazioni, volontarie e gratuite, individualmente $\mathrm{o}$ in gruppi, per le finalità di carattere sociale, civile e culturale dell'organizzazione a cui aderisce e secondo le indicazioni del Comune". Per ciò che attiene infine alle attività proposte, si promuovono generali "percorsi educativi di accoglienza e integrazione" da individuarsi "nel contesto delle attività di carattere civile e dei servizi alla comunità che non richiedono specializzazione o comunque secondo le intenzioni, capacità, professionalità, attitudini" dell'immigrato.

\section{Il discorso governativo}

Come anticipato, il clima culturale avverso sedimentatosi attorno ai richiedenti asilo ha reso evidente la necessità uno sforzo politico sul piano comunicativo, nel quale si inserisce la politica in oggetto. Ai fini di una sua totale comprensione assume pertanto grande rilievo la narrazione tramite la quale è stata promossa presso l'opinione pubblica: dall'analisi della circolare, dei

\footnotetext{
${ }^{12}$ MINISTERO DELL'INTERNO. Piano accoglienza 2016. Disponibile su: <https://www.vita.it/ attachment/d601c9b0-b314-46ba-b708-d4341546c2d9/>. Visitato il 21.12.2016.

13 Sono stati analizzati i protocolli comunali di Bergamo, Sestri Levante, Selva di Progno, Pesaro, Treviso, Padova, Terni, Vicenza e Avellino, nonché quello della Regione Emilia Romagna.
} 
protocolli prefettizi e da alcune interviste rilasciate dall'allora Ministro Alfano e dal Capo Dipartimento Morcone è stato possibile profilare il discorso governativo che ha contraddistinto il 'volontariato' dei richiedenti asilo. Riprendendo in parte la circolare 14290, dallo schema protocollare emerge il tema ambiguo dell'integrazione, anzi della "massima integrazione" che i cosiddetti "percorsi di accoglienza" tracciati tramite il 'volontariato' contribuiranno a perseguire. Concorreranno a tale obiettivo le non meglio precisate "potenzialità educativoformative" insite nelle attività, la loro capacità di facilitare la "conoscenza del tessuto sociale" dei richiedenti asilo, nonché l'apprendimento di una oscura "coscienza della partecipazione". Un ulteriore spunto interessante emerge da un secondo tema controverso, quello dell'accoglienza: finalità della politica pubblica in esame è che gli immigrati vengano "accolti anche attraverso attività e servizi resi in qualità di volontari a favore della comunità ospitante".

Dalle dichiarazioni pubbliche sul tema emerge invece una narrazione meno mistificante del 'volontariato' dei richiedenti asilo: nel maggio 2015 il ministro Alfano sdogana direttamente il termine lavoro quando esorta i Prefetti e gli enti locali a "far lavorare i migranti gratis e, ovviamente, in modo volontario, non essendo mai stato introdotto nell'ordinamento giuridico italiano il principio o l'istituto dei lavori forzati (!)"14. Conferma questa accezione il Capo Dipartimento Morcone quando in un'intervista dell'agosto del 2016 propone di superare l'esperienza del 'volontariato' coinvolgendo i profughi in attività lavorative vere e proprie, a stipendio decurtato per "recuperare $i$ costi dell'accoglienza"15. Con l'occasione riprende alcune tematiche del discorso formale: rispetto all'inattività dei profughi, sensibilizza la cittadinanza sul "rischio di lasciare queste persone appese in attesa di un destino che cada dall'alto [...] e che si abbrutiscano passando la giornata ad attendere il pranzo e la cena"; conferma il contributo che il coinvolgimento in attività lavorative potrebbero dare all'integrazione e ne evidenzia le ricadute positive in termini economici e di sicurezza; introduce infine il controverso tema della 'corsia preferenziale': "Chi mostra buona volontà (!) e capacità di inserirsi nel nostro contesto sociale potrebbe ottenere un'attenzione diversa nell'accoglienza". Ne emerge una narrazione complessiva che muove dal considerare la passività dei richiedenti asilo come ostacolo alla loro integrazione in quanto causa della rabbia popolare; per raggiungere tale agognato obiettivo, che veicola vantaggi per i profughi in termini di prospettive e per la popolazione in termini di sicurezza e ricchezza,

\footnotetext{
${ }^{14}$ FANPAGE.IT. Alfano: "Lavoro gratis per i migranti? Lo fanno già in Veneto e Toscana". 2015. Video di Youtube. Disponibile su: <https://www.youtube.com/watch?v=0KxuKw2uL-s>. Visitato il 30.12.2016.

15 IL CORRIERE DELLA SERA. II capo dell'Immigrazione Morcone: "Affidare ai profughi lavori utili". 18.08.2016. Disponibile su:<http://www.corriere.it/cronache/16_agosto_17/capodell-immigrazione-morcone-affidare-profughi-lavori-utili-e732f624-64 c4-11e-6-82810851fdf23454.shtml>. Visitato il 30.12.2016.
} 
gli immigrati vanno coinvolti in attività di 'volontariato' a favore della pubblica amministrazione, le quali daranno loro strumenti educativi, formativi, culturali e di conoscenza del tessuto sociale; i richiedenti asilo, impegnandosi in percorsi di questo tipo, saranno infine accolti con più favore dalla popolazione.

Già ad un primo livello di analisi emergono dal discorso governativo forti mistificazioni che viziano a monte la possibilità del 'volontariato' di rideterminare il clima culturale in termini di maggiore tolleranza. Si noti come vengano rimosse sia le cause reali dell'inattività dei richiedenti asilo che quelle della carenza di prospettive di integrazione, la cui natura è insita a vario titolo nel sistema politico, sociale, culturale ed economico: nel discorso governativo le due problematiche vengono invece poste in una discutibile relazione causaeffetto. Tale narrazione è però coerente con la soluzione proposta: essa implica infatti qualcosa per cui i richiedenti asilo devono impegnarsi, poiché la causa del problema viene individuata in seno alla loro condotta ed è pertanto a loro che viene attribuita la responsabilità della soluzione. Retroattivamente, individuare nei richiedenti asilo i soggetti che devono impegnarsi per emanciparsi dal presunto problema sociale e culturale della loro passività sedimenta nell'opinione pubblica l'idea che essi ne siano imputabili. Allo stesso modo si opera un ribaltamento delle responsabilità per ciò che attiene all'accoglienza: il discorso governativo ne fa emergere un concetto perlomeno inedito, inteso come 'percorso' che impegna chi è accolto, non chi accoglie. Ciò implica, in ultima istanza, una ideale divisione in seno alla categoria dei profughi, tra 'chi si dà da fare' e dimostra 'buona volontà' per farsi accogliere e chi resta ad 'abbruttirsi' nell'ozio. Rappresentata unicamente come una 'buona pratica', il 'volontariato' dei richiedenti asilo dimostra di coinvolgere piani di tipo politico, sociale e simbolico non trascurabili.

Lo studio dei casi di Torino, Selva di Progno e Treviso ha consentito di far emergere ulteriori problematiche relative alla politica del 'volontariato'. Primariamente, si delinea in via definitiva l'appartenenza di ciò che propone alla categoria del lavoro gratuito. All'interno di un panorama di forte eterogeneità delle attività progettate o implementate sono state rilevate, in primo luogo, esperienze estremamente strutturate nei tempi e nei modi di esecuzione ${ }^{16}$, in aperta contraddizione con la personalità che contraddistingue il Volontariato. Lo testimonia, in secondo luogo, il clima di aspettativa spesso osservato attorno alla loro scelta di aderire o meno ai programmi proposti: paradigmatico è il caso di Selva di Progno, nel quale il rifiuto dei profughi di proseguire l'esperienza di 'volontariato' ha scatenato le ire del sindaco.

\footnotetext{
${ }_{16}$ Tra le esperienze più strutturate sono di esempio quelle di Torino e a Vicenza, dove i richiedenti asilo sono stati integrati nelle squadre dei dipendenti delle società partecipate che si occupano di decoro urbano, pulizia e manutenzione stradale.
} 
L'esperimento ha funzionato, anche i cittadini erano felici di vederli impegnati [...] Ma la scorsa settimana, quando sono andato a proporre loro di prolungare l'esperienza per un altro mese, mi sono sentito rispondere che preferivano occupare il loro tempo per cercare un vero lavoro. Si sono fatti trascinare dai più 'sindacalizzati' del gruppo: bel modo di ricambiare l'ospitalità. ${ }^{17}$

In queste parole di condanna del primo cittadino, responsabile dell'accoglienza dei richiedenti asilo, una sorta di 'obbligo morale' sostituisce l'accezione di spontaneità che, per definizione, caratterizza il volontariato, svuotando di ogni significato questa parola in riferimento alle attività proposte.

Il clima di aspettativa permea anche le parole di alcuni amministratori quando dichiarano "Dimostrami che ti dai da fare" oppure, contestualmente ad un simile caso verificatosi presso un comune del Trevigiano, "Ci si aspettava qualcosa di più". Se dunque ai sensi della legge italiana ${ }^{18}$ si intende per Volontariato I'attività "prestata in modo personale, spontaneo e gratuito [...] senza fini di lucro anche indiretto ed esclusivamente per fini di solidarietà", ne rileva che il 'volontariato' dei richiedenti asilo non può entrare in questa categoria. II ricondurvi la policy in esame si rivela un'operazione mistificatoria che occulta attività di lavoro gratuito, del quale il 'volontariato' dei richiedenti asilo presenta caratteristiche e problematiche. Ne è un esempio la valenza pedagogica del lavoro: è in questo ambito che si intende avanzare delle ipotesi circa il contenuto delle finalità educative emerse in fase di analisi del discorso governativo. Dalle dichiarazioni di alcuni Sindaci si delinea con forza una pedagogia lavorista diretta all'educazione dei profughi ai modi ed ai tempi del lavoro occidentale, in vista di un loro disciplinamento ai ritmi e alle richieste del mercato del lavoro 'lì fuori': in una formula, lavorare gratis per imparare a lavorare. Due amministratori dichiarano:

Avevano bisogno di occupare del tempo, perché mi dicevano appunto gli operatori, siccome non avevano ritmi da fare, loro andavano a letto sempre più tardi, la mattina dormivano fino a tardi [...] Dopo qualche volta non si sono alzati, c'è anche un percorso educativo alla fine, pedagogico, 'Stamattina non ti sei alzato', ti redarguisco un attimo [...] Perché anche loro dovevano capire che c'è un caposquadra che organizzava le cose, e che nell'attività non possono fare quello che vogliono, devono stare dentro le regole.

17 IL CORRIERE DEL VENETO. Lavori socialmente utili?No Grazie Ora i profughi cercano uno stipendio. 07.09.2015. Disponibile su: <http://corrieredelveneto.corriere.it/vicenza/notizie/ cronaca/2015/7-settembre-2015/lavori-socialmente-utili-no-grazie-ora-profughi-cercanostipendio-2301886440935.shtml>. Visitato il 30.12.2016.

${ }^{18}$ Art. 2 della legge quadro n. 266 del 1999. La normativa è stata appunto elaborata per disciplinare la fenomenologia del volontariato per evitare che un abuso di tale categoria minacciasse la tutela del lavoro e dei lavoratori. MENGHINI, Luigi. Nuovi valori costituzionali e volontariato: riflessioni sull'attualità del lavoro gratuito. 
Possono avere più strumenti, imparano a confrontarsi con dei tempi di lavoro che magari per alcuni sono cose nuove... tu arrivi sul posto di lavoro, arrivi in orario? Ci sono delle regole quando vai a lavorare, come quando vai a scuola [...] Dice questi sono fissati, però la nostra società funziona anche con queste regole.

Dalle dichiarazioni emerge anche una specifica 'pedagogia del lavoro gratuito'. In primo luogo, il 'volontariato' dei richiedenti asilo ne replica l'impatto sull'autorappresentazione del soggetto come (non) lavoratore e del lavoro stesso come (non) lavoro: I'etichettamento di una prestazione lavorativa con la dicitura 'volontariato' opera la stessa mistificazione che si verifica per altre attività gratuite, ad esempio il tirocinio. Al pari degli stagisti, i richiedenti asilo sono portati a non concepire il loro lavoro come tale, con la medesima conseguenza di unthink work, e quindi di unthink exploitation ${ }^{19}$. L'unpaid work modella inoltre le aspettative di chi fa il suo ingresso nel mondo del lavoro: se nel caso dei tirocinanti "it undervalu[es] their stock value so that they willingly and competitivaly take up the 'opportunity' on an unpaid placement to increase their value in the future ${ }^{\prime 20}$, una medesima operazione si può immaginare per i richiedenti asilo, ai quali viene indiscriminatamente proposta una attività gratuita e non professionalizzante: assieme al regime stesso delle migrazioni, che rende più difficile (ma non blocca) i movimenti delle popolazioni dalle periferie al centro del mondo neo-globalizzato, il lavoro gratuito ne abbassa le aspettative e crea i presupposti per la formazione di una classe lavoratrice più flessibile ed economica.

Se dunque dalle parole degli amministratori si intuisce la convinzione di un portato educativo del 'volontariato', controversamente declinato come disciplinatorio e veicolante peraltro un'utilità stessa per i profughi in quanto potenziali futuri lavoratori ("Vengono da mondi molto diversi quindi anche solo il fatto di arrivare in orario, presentarsi, avere una regolarità per loro è molto importante..."), esso si rivela essere per altri aspetti del tutto inutile in relazione ad altri obiettivi espressi dal discorso governativo. Per ciò che attiene ad esempio alle finalità formative, il censimento delle attività implementate non può che smentire l'esistenza di qualsiasi possibilità di apprendimento professionale: alcuni intervistati insistono sull'insignificanza delle stesse da un punto di vista pratico, connotandole ripetutamente con gli aggettivi "piccolo, piccolissimo" o "minimo". II 'volontariato' si è rivelato inutile anche relativamente agli obiettivi della partecipazione e della conoscenza del tessuto sociale. L'orientamento nella città e la creazione di relazioni in loco che favoriscano il reciproco avvicinamento tra cittadini e richiedenti asilo

\footnotetext{
${ }^{19}$ RODINO-COLOCINO, BERBERICK, op. cit., p. 491.

${ }^{20}$ HOPE, FIGIEL, op. cit., p. 369. Sul tema: SMITH, Vicki. Review article - Enhancing employability: Human, cultural, and social capital in an era of turbulent unpredictability.
} 
sono prerogative riconosciute da molti intervistati come fondamentali per il miglioramento della qualità della vita e delle prospettive di permanenza dei profughi sul territorio. Tuttavia, la strutturazione stessa dell'attività favorisce nulla più di ciò che un amministratore ha definito "contatto indiretto" tra immigrati e autoctoni, una non-relazione basata su un'attività che nemmeno sulla carta prevede uno scambio reale e paritario. Le relazioni effettivamente create sono risultate minime e limitate spesso al singolo volontario che si è incaricato di seguire la squadra di immigrati nelle pulizie, nonché ostacolate dalla frequente non conoscenza di altre lingue oltre all'italiano da parte di chi si è messo a disposizione. Eppure l'inchiesta stessa ha evidenziato come le relazioni tra persone, e percorsi di partecipazione che le implichino e facilitino, siano I'unica soluzione per una inclusione de facto dei richiedenti asilo. In questo senso il Volontariato costituisce uno strumento prezioso per coinvolgere gli immigrati nel tessuto sociale delle comunità nonché contribuire a risolvere le problematiche connesse al loro isolamento e al fallimento della terza accoglienza ${ }^{21}$. Alcuni intervistati testimoniano a tal proposito della creazione di reti sociali all'interno delle quali alcuni richiedenti asilo hanno trovato ospitalità una volta usciti dai centri, o addirittura un'occupazione. Di certo le carenze e criticità connesse all'accoglienza sono sistemiche e la soluzione non deve essere ricercata nel Volontariato, del quale non si intende fare alcuna apologia: le sue crescenti interazioni con un settore pubblico sempre più sottile portano con sé ambiguità e problematiche. Si ritiene tuttavia che, nell'ottica di una fruttuosa quanto indispensabile partecipazione dei richiedenti asilo al tessuto sociale delle comunità ospitanti, il Volontariato possa e debba sostituire il 'volontariato' nelle agende degli amministratori locali mettendo in campo i suoi valori originali: creare ponti tra le persone e diffondere pratiche di solidarietà. In questo si sostanza una possibile via partecipativa all'integrazione, senza dubbio più promettente di quella caratterizzata dal lavoro dequalificato, gratuito o sottopagato proposta a livello ministeriale. Quest'ultimo è per vari aspetti di tipo normativo: oltre al portato pedagogico discusso precedentemente, emerge in quest'ambito il fondamentale ruolo della Prefettura in quanto responsabile dell'accoglienza straordinaria. Dettando le regole all'interno delle quali si determina la quotidianità della maggior parte dei richiedenti asilo, essa ha potere decisionale anche rispetto alle attività che essi possono svolgere: tra queste, quelle del 'volontariato' sono molto caldeggiate, mentre per altre non viene concesso il nullaosta. II protagonismo delle Prefetture determina dunque una gestione eterodiretta della presenza dei profughi sui territori, nella quale le preponderanti prerogative di controllo governative rendono l'obiettivo della

${ }_{21}$ Per 'terza accoglienza' si intende la fase successiva all'uscita dei richiedenti asilo dai centri di accoglienza una volta riconosciuta loro la protezione internazionale, per la quale si sta verificando un silenzioso disinteresse da parte della politica. 
partecipazione, I'unico realmente utile ai richiedenti asilo tra quelli formalmente proposti nel discorso governativo, mera retorica.

Contrapponendosi all'inutilità de facto delle attività proposte, emerge come monumentale paradosso il ricorrente tema dell'utilità che, stando alle dichiarazioni raccolte, i richiedenti asilo dovrebbero dimostrare ad un'opinione pubblica che li accusa di costituire un mero peso per l'economia. Tramite il 'volontariato' è richiesto ai richiedenti asilo di rendersi utili con lo strumento legalmente e socialmente richiesto agli altri immigrati come condizione per la loro permanenza sul territorio nazionale, ossia il lavoro: dando il loro "contributo agli occhi della cittadinanza" dimostrano che, come gli altri immigrati, sono anch'essi utili alla società e quindi meritevoli di restare. Ma se dunque ai profughi è diretta una massiccia richiesta di rendersi utili tramite un'attività inutile, a cosa è utile il 'volontariato' dei richiedenti asilo?

[È un] progetto molto generico, volutamente generico, nel quale fossero previste attività semplici, che però fossero percepite anche a livello della cittadinanza come utili. E soprattutto che servissero a creare quell'attenzione e atteggiamento positivo della cittadinanza nei confronti di queste persone. Superare la diffidenza, i timori... la via più facile è quella di capire che queste persone si impegnano in attività che poi sono di utilità comune. [...] C'è una realtà percepita, purtroppo bisogna dirlo, che ha la precedenza sulla reale consistenza di un fenomeno.

Le paradigmatiche parole di questo Sindaco consentono di cogliere che I'utilità che i richiedenti asilo dovrebbero dimostrare attraverso il 'volontariato' non è concepita come reale, materiale (essi fanno qualcosa per la comunità) ma è giocata a livello di percezione: gli immigrati fanno vedere che fanno qualcosa per la comunità. Nei colloqui si ripetono con estrema frequenza termini come vedere, di-mostrare, rendere evidente, esporre, percepire, (far) capire: ai fini del farsi accogliere non è importante ciò che i richiedenti asilo fanno, ma che facciano vedere che fanno.

[Bisogna] rendere evidente che non è che tutto il giorno non fanno niente, allora c'è anche una partecipazione pubblica in cui in qualche modo, se la comunità accoglie lo straniero...

Un territorio non si apre da solo alla visione anche di persone diverse da noi, anche solo nel colore della pelle [...] Intanto la visibilità... infatti quello che noi abbiamo pensato da subito è che questi ragazzi andassero in giro, fossero visibili, che fossero visti insomma, non sono dei mostri, possono fare delle cose utili per tutti.

È nel messaggio ai fini del consenso che risiedono le reali motivazioni politiche del 'volontariato' dei richiedenti asilo. A quest'ultimi viene riservato il ruolo di attori passivi in una politica pubblica che rivela di non considerare alcuna delle loro attuali e future esigenze, ma risulta utile alla necessità della 
classe politica di consegnare all'irritata popolazione una risposta superficiale ad un presunto problema, quello dell'inattività dei profughi, rispetto alla quale le domande sono peraltro malposte e i responsabili individuati nelle vittime stesse di un sistema inefficiente e corrotto.

L'inchiesta ha però evidenziato come la risposta governativa non sia solo superficiale, ma veicoli anche potenziali conseguenze negative. Assumendo la prospettiva per cui l'efficacia del 'volontariato' si gioca sulla percezione di utilità dei richiedenti asilo e che esso sia pertanto impostato per avere un impatto sulla costruzione dell'immaginario collettivo, emerge l'importanza del tipo di attività prevista dalla politica pubblica. È innegabile che la pulizia delle strade e la raccolta dei rifiuti siano mansioni dignitose ma molto caratterizzanti: il fatto che ai profughi sia proposta un'attività gratuita ed 'in vetrina' di questo tipo contribuisce a sedimentare un immaginario collettivo per il quale è considerato normale assegnare automaticamente agli immigrati i 3D-jobs, "dirty, dangerous, demanding" ${ }^{\prime 22}$. Se unitamente a ciò si considera una delle polemiche collegate all'inattività dei profughi, quella del loro 'bighellonare' (ossia il loro frequentare gli spazi pubblici senza un apparente - dal punto di vista degli autoctoni motivo), il 'volontariato' tende a trasformare lo spazio della città in un luogo che i profughi possono frequentare, nel duplice senso di possibilità reale e socialmente accettata, solo nei tempi e nelle attività rigidamente regolamentati del lavoro gratuito di pubblica utilità. Si ritiene pertanto che vedere all'opera gruppi di richiedenti asilo, una categoria sotto la luce dei riflettori popolari e mediatici, impegnati gratuitamente nella pulizia degli spazi delle città che essi stessi non praticano (o non praticano liberamente) contribuisca a sedimentarne un'immagine inferiorizzata. II 'volontariato' rischia di avere delle implicazioni anche sullo stesso concetto socialmente condiviso di diritto d'asilo e sulla sua, pur formale, universalità. In primo luogo, il suggerimento di legare l'accoglienza dei richiedenti asilo ad una dimostrazione di 'buona volontà', rispetto alla quale il 'volontariato' rappresenta uno strumento, introduce l'opinione pubblica ad una cognizione di diritto d'asilo populista ed estranea a qualsiasi normativa internazionale. Dividendo sulla base di un criterio impostore i profughi in due categorie ne mina il fondamentale carattere di universalità e ne deforma il principio chiave per cui l'assistenza legata alla richiesta d'asilo va fornita a chiunque ne faccia istanza, a prescindere che se la guadagni attraverso il 'volontariato' o altre forme di impegno. Questa narrazione implica peraltro un condizionamento dell'idea collettiva rispetto alla natura stessa del diritto d'asilo. Per argomentare questo passaggio è utile muovere dal tema della restituzione: del tutto assente nelle comunicazioni ufficiali, la necessità che i richiedenti

${ }_{22}$ PIORE, Michael. Birds of passage. New York: Cambridge University Press, 1979, p. 29. 
asilo si sdebitino restituendo prende forma più o meno netta nelle parole dei Sindaci. Un amministratore dichiara:

[E' necessario] far capire a questi richiedenti asilo che loro sono qua, ospitati da questo Paese, e che fa un investimento di denari per questa loro presenza qua, e che voi restituirete qualcosa a questo territorio se questo territorio ti da mangiare, da dormire e ti da un tetto [...] Per loro [cittadini], tu sei qua ospite di questo territorio, pagato, spesato, alloggiato da questa nazione, devi restituire a questo territorio qualcosa, no? In termini di lavoro, perché sei ospitato. L'altro è di dire al nostro territorio, ai nostri concittadini che quelli che sono ospito qua non sono solo a spese della comunità, ma restituiscono anche alla comunità dei lavori $[. ..] \mathrm{Ci}$ sono due aspetti da considerare... uno è quello immediato. Tu sei qui, in questo momento, non sappiamo se resti su questo territorio, ma finché resti restituisci qualcosa.

II caso di Torino si erge a paradigma del concetto di restituire per farsi accogliere. L'allora sindaco Piero Fassino descrive il 'volontariato' come "una forma di restituzione, su base volontaria, per l'accoglienza che Torino ha dato loro [...] II fatto che ci siano azioni di restituzione come questa aiuta a favorire I'accettazione dell'accoglienza da parte della popolazione"23; per sedimentare il concetto ha dotato i richiedenti asilo di casacchine catarifrangenti che recano sul dorso la scritta "GRAZIE TORINO". Questa consolidata e mediaticamente amplificata convinzione implica la costruzione di un senso comune relativo al diritto d'asilo nel quale il piano inclinato dello sdebitarsi restituendo lo fa scivolare dal paradigma dei diritti universali a quello delle concessioni statali. In quanto strumento per farsi accogliere, la restituzione tramite il 'volontariato' assume un carattere simbolico per il quale, più che gli altri immigrati, ai richiedenti asilo viene affidato un ulteriore fardello, una sorta di colpa da espiare, ossia quella di essere accolti a spese dello Stato. Sono chiamati a di-mostrare un surplus di impegno gratuito per ambire ad un'inclusione... subordinata ${ }^{24}$ e precaria ${ }^{25}$, come quella sperimentata dagli altri immigrati in Italia. Si tratta di una sorta di espiazione funzionale al concetto rovesciato di accoglienza discusso precedentemente, nel quale la responsabilità della costruzione di una convivenza virtuosa e pacifica ricade su chi necessita di essere accolto e non su chi accoglie.

Per chiudere la dissertazione, è necessario ritornare sul ruolo dei Comuni nel sistema d'accoglienza: è infatti dalla lettura del 'volontariato' in

\footnotetext{
${ }^{23}$ LA REPUBBLICA - EDIZIONE DI TORINO. I profughi puliscono Torino: per tre mesi 27 rifugiati spazzini volontari. 23.04.2015. Disponibile su: <http://torino.repubblica.it/ cronaca/2016/04/23/news/i_profughi_puliscono_torino_per_tre_mesi_27_rifugiati_spazzini_ volontari-138276867/>. Visitato il 30.12.2016.

${ }^{24} \mathrm{DE}$ GIORGI, Alessandro. L'Europa fra stato penale e nuova cittadinanza. In BRONZINI, Giuseppe (a cura di). Europa, costituzione e movimenti sociali. Roma: ManifestoLibri, 2010, p. 251.

${ }^{25}$ PEROCCO, op. cit., p. 136.
} 
relazione alle sfide poste dall'immigrazione alle amministrazioni locali che se ne intuisce la funzione pragmatica. Se è ipotizzabile che la politica pubblica in esame influenzi (negativamente) l'immaginario collettivo attorno alla tematica dei richiedenti asilo, non è a livello comunale che va imputata questa responsabilità: attualmente i Comuni si trovano 'tra due fuochi', nella spinosa posizione di mediatori tra il Governo che 'smista' i richiedenti asilo in assenza di un piano concertato con i territori ed una popolazione nutrita per decenni con il pane dell'intolleranza e della paura. È in questo contesto che il 'volontariato' dei richiedenti asilo assume la funzione di una 'pillola' somministrata alla comunità per far andar giù il boccone amaro dell'accoglienza. Obiettivo del presente studio è stato il far emergere le numerose e gravi controindicazioni ad essa associata, le quali rischiano di affliggere un immaginario collettivo già gravemente affetto da disinformazione e stereotipi.

\section{Conclusioni}

La presenza di un clima di intolleranza popolare nei confronti dei richiedenti asilo in Italia è una realtà inconfutabile, che trova legittimamente spazio nell'agenda politica e contro la quale vanno messe in campo urgenti ed incisive politiche pubbliche. Per sperimentare delle virtuose formule di convivenza tra autoctoni e immigrati vanno però individuati e messi in discussione le cause delle criticità insite nel sistema di accoglienza nonché i reali responsabili dell'intolleranza: la disinformazione, il razzismo istituzionale e mediatico, le diseguaglianze sociali. Mettendo in luce le numerose un mistificazioni e contraddizioni che contraddistinguono il discorso governativo tramite il quale il 'volontariato' dei richiedenti asilo è stato pubblicamente promosso, lo studio ha voluto dimostrare come esso si muova nella direzione opposta. È infatti improbabile creare un clima più favorevole alla presenza dei richiedenti asilo imputando, pur indirettamente, le responsabilità dell'emergenzialità della situazione agli immigrati stessi, nonché consegnando alla loro 'buona volontà' la possibilità di farsi accogliere. Si è dimostrato come il 'volontariato' dei richiedenti asilo abbia un impatto negativo sull'immaginario collettivo: giocando la sua efficacia su un piano percettivo orientato al di-mostrare una simbolica utilità sociale dei profughi attraverso lo svolgimento di mansioni dequalificate, esso ne sedimenta un'immagine inferiorizzata e prescrive loro una modalità estremamente vincolante di frequentazione degli spazi pubblici. In secondo luogo, tende a sedimentare la pericolosa ed infondata idea di un non-diritto alla protezione internazionale e all'accoglienza: I'universalità del diritto d'asilo viene inficiata dall'ideale divisione tra richiedenti asilo 'meritevoli' di accoglienza e 'inutili nullafacenti' che il 'volontariato' opera quando pone nelle loro mani la responsabilità di farsi accogliere. Il lavoro gratuito, oltre ad educare i richiedenti asilo ad un'inclusione subordinata nel mondo del lavoro, 
in quanto strumento di restituzione dell'assistenza costituisce infine la base di una sorta di un'espiazione spettacolarizzata tramite la quale si intende dare ai richiedenti asilo l'occasione di-mostrare 'buona volontà' per meritare l'accoglienza tramite la cura materiale di un territorio dal quale si è di fatto esclusi. Ne emerge in ultima analisi una politica pubblica dai risvolti a dir poco paradossali: narrata come strumento elaborato per migliorare la convivenza tra popolazione autoctona e richiedenti asilo, essa si rivela dannosa nei loro confronti in quanto li inferiorizza e mette in discussione il loro stesso diritto di essere accolti.

II 'volontariato' si allinea dunque alle politiche ed i discorsi che allontanano la prospettiva di una più serena e proficua convivenza tra richiedenti asilo e popolazione autoctona, più che mai necessaria a causa del, e nonostante il, contesto di crisi economica e sociale che l'Italia e l'Europa intera stanno attraversando. Le recenti scelte di politica pubblica del Governo italiano sembrano tuttavia confermare la tendenza che il 'volontariato' ha contribuito a tracciare: accanto a narrazioni pubbliche persistentemente mistificanti, nel Piano Accoglienza 2017 il Governo ha annunciato di voler introdurre il vincolo tra espletamento di lavori socialmente utili e riconoscimento della protezione internazionale, confermando una tendenza di istituzionalizzazione del lavoro gratuito o sottopagato dei richiedenti asilo. II presente studio ha voluto invece contribuire a dimostrare la necessità e la possibilità di ideare, promuovere e implementare politiche pubbliche che abbiano l'obiettivo concreto di far partecipare e non di far vedere, di creare relazioni e non di simularle, per favorire una virtuosa e reale inclusione dal basso dei richiedenti asilo.

\section{Bibliografia essenziale}

CHARMAZ, Kathi. Constructing grounded theory. Los Angeles: Sage, 2014.

HOPE, Sophie; FIGIEL Joanna. Interning and investing: Rethinking unpaid work, social capital, and the "Human capital regime". TripleC, v. 13, n. 2, 2015, p. 361-374.

MAES, Kenneth. Volunteerism or labor exploitation? Harnessing the volunteer spirit to sustain AIDS treatment programs in urban Ethiopia. Human organization, v. 71, n. 1, 2012, p. 54-64.

MENGHINI, Luigi. Nuovi valori costituzionali e volontariato: riflessioni sull'attualità del lavoro gratuito. Milano: Giuffrè, 1989.

PEROCCO, Fabio. Trasformazioni globali e nuove disuguaglianze. Milano: Franco Angeli Editore, 2012.

RODINO-COLOCINO, Michelle; BERBERICK Stephanie Nicholl. "You kind of have to bite the bullet and do bitch work". How internships teach students to unthink exploitation in public relations. TripleC, v. 13, n. 2, 2015, p. 486-500. 
SMITH, Vicki. Review article - Enhancing employability: Human, cultural, and social capital in an era of turbulent unpredictability. Human Relations, v. 63, n. 2, 2010, p. 279-300.

STRAUSS Anselm; CORBIN Juliet. Basics of qualitative research. Newbury Park, CA: Sage, 2010.

Articolo ricevuto il 31.01.2017 Accettato per la pubblicazione il 06.03.2017 Received for publication in January 315t, 2017 Accepted for publication in March 06 ${ }^{\text {th }}, 2017$

ISSN impresso 1980-8585 ISSN eletrônico 2237-9843 http://dx.doi.org/10.1590/1980-85852503880004913 